\title{
Development of Azithromycin Ophthalmic Gels Using Various Polymers
}

\section{Harith Salam Mohammed, Ahmad Makame Mwadini, Nida'a Mohammed Ali Wadi and Alka Ahuja*}

College of Pharmacy, National University of Science and Technology, Muscat, Sultanate of Oman, Oman

*Corresponding Author: Alka Ahuja, Professor of Pharmaceutics, College of Pharmacy, National University of Science and Technology, Oman.
Received: February 27, 2020

Published: March 17, 2020

(C) All rights are reserved by Alka Ahuja., et al.

\begin{abstract}
Different formulations for the treatment of eye infections are usually administered in the form of conventional ocular drug delivery systems which are topical eye drops or ointments (1). Typically, ophthalmic bioavailabilities of only $1-10 \%$ are achieved due to the short precorneal residence time of ophthalmic solutions. (2) The preparation and evaluation of gel containing antibiotic azithromycin combined with different polymers like Carbopol, sodium alginate and Hydroxypropyl cellulose (HPC) was done and assessed to find out which polymer could best be used in preparing ophthalmic gels for this antibiotic using factorial design. Since the efficacy of these gels is dependent on factors like viscosity and $\mathrm{pH}$, the polymers in these gels were also examined for different parameters such as $\mathrm{pH}$, in vitro release, permeation and microbiological evaluation.
\end{abstract}

Keywords: Drug; Ophthalmic Gels; Eye Drops

\section{Introduction}

Several ophthalmic problems have been investigated including bacterial conjunctivitis, blepharitis and keratitis, and topical antibiotics have been used in the treatment of these problems. Different formulations for treatment of eye infections are usually administered in the form of conventional ocular drug delivery systems which are topical eye drops or ointments [1]. Eye drops and suspensions are often used for topical administration of ophthalmic active drug to tissue around ocular cavity.

The limitation of the conventional liquid ophthalmic formulations is that they demonstrate low bioavailability because of constant lacrimal drainage in the eye. The normal drainage of an instilled drug dose commences immediately upon instillation and is essentially completed within 5 min. Typically, ophthalmic bioavailability of only $1-10 \%$ are achieved due to the short precorneal residence time of ophthalmic solutions [2].

Consequently, there is a need for frequent instillation of concentrated solutions to achieve the desired therapeutic effect, which may lead to some undesirable side effects. Many techniques are applied to prolong the drug stay at site of action and attain optimal concentration. The use of gels for ocular administration of drug offers many advantages compared to conventional ocular drug delivery systems like eye drops which leads to prolonged corneal contact time [3].

Jain SP., et al. (2008) prepared and evaluated ophthalmic delivery system for ciprofloxacin hydrochloride based on the concept of $\mathrm{pH}$-triggered in situ gelation. The in situ gelling system involved the use of Polyacrylic acid (Carbopol 980) as a phase transition polymer, hydroxypropyl methylcellulose as a release retardant, and ion exchange resin as a complexing agent. The developed formulation was stable and in vitro drug release was found to be around $98 \%$ over a period of 24 hours [4].

Ma WD., et al. investigated the in vitro drug release of Pluronic acid copolymer gels, as well as the in vivo resident properties of in situ gel ophthalmic formulations. These in vivo experimental results demonstrated that in situ gels containing Pluronic acid copolymer may significantly prolong the drug residence time and thus improve bioavailability [5].

Al-Kassas RS., et al. also performed a study to design controlled release ophthalmic delivery systems for ciprofloxacin based on polymeric carriers in an attempt to prolong the effect of ciprofloxacin and improve its ocular bioavailability. Carbopol and alginates polymers were used to confer gelation properties to the formulations. Controlled release formulated gels consisting of carbopol and cellulose derivatives showed an increase in viscosity, gelling capacity and adhesiveness as the concentration of each polymeric component was increased [6].

Vyas SP., et al. described ophthalmic delivery system of an antiglaucoma drug, timolol maleate (TM) based on the use of polymers. The results clearly demonstrated that developed carbopol-chitosan based formulation was therapeutically efficacious and showed a diffusion controlled release behaviour over $24 \mathrm{~h}$ periods [7].

Padma Preetha J., et al. formulated and evaluated in-situ gels of Diclofenac sodium. These formulations were prepared by combination of HPMC, HPC, HEC and Carbopol derivatives. They performed the studies such as drug content, $\mathrm{pH}$ of the Ophthalmic gels, invitro diffusion study, sterility testing, anti-microbial studies, antibacterial and anti-fungal studies. The percentage cumulative drug 
release of the best formulation was $87.23 \%$ compared to other formulations for the period of 8 hours. The selected formulations showed the antimicrobial, antibacterial and antifungal efficacy [8].

Kesavan K., et al. studied HP-p-CD based mucoadhesive hydrogel for ophthalmic delivery of Dexamethasone (DXN) to treat uveitis and improve ocular retention and sustained action. The developed HP-P-CD-based mucoadhesive system was alternative to conventional eye drops of DXN due to its ability to enhance bioavailability through its longer precorneal residence time and ability to sustain the release of the drug [9].

Wu H., et al. investigated the correlation between the stability of baicalin and drug release. Carbopol 974P (0.3\%, w/v) was used as the gelling agent combined with Hydroxypropyl methylcellulose E4M $(0.6 \%, \mathrm{w} / \mathrm{v})$ which acted as a viscosity enhancing agent. The results demonstrated that ophthalmic gels have better ability to keep baicalin stable and retain drug release than marketed baicalin eye drops. Gels had enhanced the ocular bioavailability [10].

Piyush B. Patel designed a study to optimize and characterize an ophthalmic gel of an antibacterial agent, ofloxacin using Carbopol as the gelling agent along with Polycarbophil as viscosity enhancer. A 32 factorial design was applied for the optimization of the final formulation. The optimized formulation was found to be stable, therapeutically efficacious and provided sustained release of the drug over a $8 \mathrm{~h}$ period [11].

Lokhande Umesh Ramachandra., et al. designed and developed a pH-triggered in-situ gelling system of Ciprofloxacin using Carbopol and HPMC. The poor bioavailability and therapeutic response exhibited by conventional ophthalmic solutions due to rapid precorneal elimination of the drug was overcome by the use of in-situ gel forming systems that are instilled as drops into eye and undergo sol-gel transition in the cul-de-sac. The in-vitro release studies showed the release as $97.33 \%$ for the period of 8 hours [12].

Nisha Shetty G., et al. formulated Naphazoline and antazoline in-situ gelling systems for ocular delivery and carried out stability studies. The polymers used for preparation of in situ gel were Carbopol 940 and HPMC K4M. The formulations were evaluated by using their $\mathrm{pH}$, Isotonicity, gelling capacity, rheological characteristics, in-vitro release, sterility and in-vivo studies. Their formulations were proved to be very stable at room temperature and at higher temperature $\left(40^{\circ} \mathrm{C}\right)$ with satisfactory drug release [13].

Nnamani PO designed the study for the formulation of ocular gels based on polymer Carbopol to evaluate suitability for enhanced topical delivery of aminoglycoside antibiotic gentamicin. The formulated gels were evaluated in terms of drug content, $\mathrm{pH}$, physical assessment, viscosity, stability as well as drug release. The study demonstrated that gels of gentamicin made with polymer may offer a promising approach for topical delivery of gentamicin for the treatment of infections caused by gentamicin-susceptible bacteria [14].
Prachi Saxena., et al. (2014) developed the pH sensitive hydrogels of Levofloxacin hemihydrates for ophthalmic drug delivery using Carbopol 940 and HPMC. Levofloxacin is widely used for the treatment of acute conjunctivitis. The drug suffers the drawbacks of poor bioavailability due to its $\mathrm{pH}$ dependent solubility. Evaluation parameters were clarity, drug content, gelling capacity, viscosity studies and in vitro release studies. In-vitro release revealed that best formulation showed the release of $84.31 \%$ [15].

Prolongation of residence time due to these ophthalmic gels helps to deliver a drug continuously in a controlled manner to the anterior chamber of the eye and eliminates frequent administration of the drug, thus leading to better patient compliance and extended action. This results in a dose reduction and helps to minimize local and systemic side effects. The preparation and evaluation of gel containing antibiotic azithromycin combined with different polymers like Carbopol, sodium alginate and Hydroxypropyl cellulose (HPC) was done and assessed to find which polymer could best be used in preparing ophthalmic gels for this antibiotic using factorial design. Since the efficacy of these gels is dependent on factors like viscosity and $\mathrm{pH}$, the polymers in these gels were also examined for different parameters such as $\mathrm{pH}$, in vitro release, permeation and microbiological evaluation.

In the present study ophthalmic gels were prepared for Azithromycin using various polymers and evaluated. Azithromycin, 1-oxa-6-azacyclopentadecan-15-one, 13-[(2,6-dideoxy-3-C-methyl-3-0 -methyl- $\alpha$-L-ribo-hexopyranosyl)oxy]-2-ethyl-3,4,10-trihydroxy-3,5,6,8,0,12,14-heptamethyl-11-[3,4,6-trideoxy-3(dimethylamino)- $\beta$-D-xylo-hexopyranosyl)oxy], dihydrate, an azalide is , a subclass of macrolide antibiotics. The macrocyclical lactone expansion and the presence of nitrogen atom in the lactone ring are responsible for the best pharmacological and microbiological characteristics. Azithromycin has similar antimicrobial spectrum as erythromycin, but is more effective against certain gramnegative bacteria, particularly Haemophilus influenzae.

Azithromycin prevents bacteria from growing by interfering with their protein synthesis. Azithromycin binds to the $50 \mathrm{~S}$ subunit of the bacterial ribosome, and thus inhibits translation of mRNA but nucleic acid synthesis is not affected. It can be used to treat ophthalmic bacterial infections like bacterial conjunctivitis. Azithromycin is used widely in clinical practice and recently it is available in topical solution for ophthalmic use. Topical azithromycin was found effective and safe in various ocular surface infections, in meibomian gland dysfunction and in trachoma. Also, it may substitute fluoroquinolones in corneal UV cross-linking. Azithromycin is one of the safest antibiotics, well tolerated, and with special pharmacokinetic properties. Also, it is characterized by a broad antimicrobial spectrum. Azithromycin is efficacious for the treatment of a lot of ocular diseases and may be included as monotherapy or in combination therapy in new treatment protocols for more ocular infections. However, more research is needed to determine this. Hence, to prolong its residence time and better action, it was planned to formulate it as ophthalmic gels [16]. 


\section{Aim and Objectives of the Study}

Aim

The aim of the study was to formulate and evaluate the ophthalmic gels of azithromycin using different polymers and find out which polymer is best suited in preparing ophthalmic gels for the antibiotic gel. Conventional Ocular Drug Delivery Systems used as topical eye drops or ointments are the most common formulations used for ophthalmic infections. Major limitation of these formulations is the low bioavailability of the drug because of constant lachrymal drainage, resulting in short precorneal residence time of ophthalmic solutions and need for frequent instillation to achieve the desired therapeutic effect. Use of gels for ocular administration of drug offer many advantages as they prolong corneal contact time and improve bioavailability leading to better patient compliance, extended action, decrease in frequency that helps in minimizing systemic side effects. In the present work, the aim was to prepare antibacterial azithromycin gel using polymers Carbopol 940 and hydroxypropyl cellulose as gelling agents and assess using $3^{2}$ factorial design.

\section{Objectives}

The objectives of the present study were

- To prepare ocular gels using different polymers like Carbopol 940 and Hydroxypropyl Cellulose (HPC)

- $\quad$ To evaluate the prepared formulations for parameters like $\mathrm{pH}$, in vitro release, antimicrobial activity and drug permeation.

\section{Materials and Methods}

\section{Materials}

- Chemicals: Carbopol 940P was gifted by Hangzhou Lingeba technology Co. Ltd. Hydroxypropyl Cellulose (HPC) and Azithromycin were gifted by National Pharmaceutical Industries (NPI) Oman. All other chemicals including sodium chloride, hydrated calcium chloride, sodium carbonate, sodium hydroxide were taken from College of Pharmacy Laboratory.

- Equipment's: Beakers, measuring cylinders, conical flasks, reagent bottles, glass rods, watch glasses.

- Apparatuses: Dissolution apparatus (veego), UV-spectrophotometer (6705 UV/Vis. Spectrophotometer by Jenway), pH meter (Martini by Milwaukee Instrument Company) and Franz apparatus (fabricated).

\section{Methods}

\section{Factorial design}

Optimization was done using $3^{2}$ full factorial design in which two factors were evaluated and experimental trials were performed on all 9 possible combinations. In this design there were two independent variables and 3 levels which were low, medium, and high of each variable applied.

The advantages of a factorial design include greater precision. Using a factorial design allows examination of the effect of one variable when other factors are changed, something which is not possible using traditional methods of investigation. Two different polymers namely Cabopol and HPC were chosen for the study. Carbopol was used in three different concentrations whereas \% of HPC was kept same. The formulae are given in Table 1.

\section{Preparation of ophthalmic gels}

Composition of carbopol (940) gel

Formulations were prepared using a $3^{2}$ factorial design (Table 1). The weighed amount of Carbopol was added in the beaker with a little amount of water and then stirred using glass rod to dissolve the added Carbopol. Hydroxypropyl Cellulose (HPC) was then added to previous formulation and water was added and stirred. Azithromycin was finally added with remaining amount of water to make volume up to $50 \mathrm{ml}$. To provide alkaline medium for the swelling of Carbopol to form gel, $0.5 \mathrm{~N} \mathrm{NaOH}$ was added drops-wise until gel was formed.

\begin{tabular}{|l|c|c|c|}
\hline $\begin{array}{c}\text { Formulation } \\
\text { Code }\end{array}$ & $\begin{array}{c}\text { Azithromycin } \\
\mathbf{\% w} / \mathbf{w}\end{array}$ & $\begin{array}{c}\text { Carbopol } \\
\mathbf{\% w} / \mathbf{w}\end{array}$ & HPC \%w/w \\
\hline Gel 1 & 0.5 & 0.25 & 0.5 \\
\hline Gel 2 & 0.5 & 0.25 & 1.0 \\
\hline Gel 3 & 0.5 & 0.25 & 1.5 \\
\hline Gel 4 & 0.5 & 0.375 & 0.5 \\
\hline Gel 5 & 0.5 & 0.375 & 1.0 \\
\hline Gel 6 & 0.5 & 0.375 & 1.5 \\
\hline Gel 7 & 0.5 & 0.5 & 0.5 \\
\hline Gel 8 & 0.5 & 0.5 & 1.0 \\
\hline Gel 9 & 0.5 & 0.5 & 1.5 \\
\hline
\end{tabular}

Table 1: Composition of ophthalmic gels using Carbopol 940P.

\section{Evaluation of gels}

- Physical appearance: The formulated gels were observed for clarity, homogeneity and presence of bubbles in them.

- $\quad$ H evaluation: $1 \mathrm{ml}$ of gel was added in beaker and $10 \mathrm{ml}$ of water was added and stirred completely. $\mathrm{pH}$ was measured by digital $\mathrm{pH}$ meter in triplicate to get mean and standard deviation.

- In vitro release: The drug release from different Azithromycin containing polymer gels was studied using dissolution testing apparatus type $1.1 \mathrm{ml}$ of the gel was filled inside cellulose bag using $2.5 \mathrm{ml}$ syringe and kept inside the basket of the dissolution apparatus. The container was immersed in $700 \mathrm{ml}$ freshly prepared simulated tear fluid $(\mathrm{NaCl} 6.7 \mathrm{~g}$, $\mathrm{NaHCO}_{3} 2 \mathrm{~g}, \mathrm{CaCl}_{2} 0.08 \mathrm{~g}$ and distilled water up to $1000 \mathrm{ml}$ ) with adjusted $\mathrm{pH}$ at 6.85 , which was used as dissolution medium. Temperature of $37 \pm 0.50^{\circ} \mathrm{C}$ and rotations of $100 \mathrm{rpm}$ were maintained throughout the test. Aliquots of $5 \mathrm{ml}$ were withdrawn from release medium at each sampling time and replaced with an equal volume of the prepared buffer. The drug content in the samples was determined at $240 \mathrm{~nm}$ using UV-Visible spectrophotometer. 
- Preparation of standard curve for the release and permeation study: The stock solution of $20 \mu \mathrm{g} / \mathrm{ml}$ was prepared using the drug and methanol. $2 \mathrm{mg}$ of azithromycin was weighed and dissolved in methanol and made up to $100 \mathrm{ml}$ using volumetric flask. The solution was stirred well using magnetic stirrer to get uniform solution. Then six dilutions of concentrations 2,4,8,10,16 and $20 \mu \mathrm{g} / \mathrm{ml}$ were made from stock solution and made up to $10 \mathrm{ml}$. These dilutions were finally used for the plotting of standard curve. Calibration of UV-spectrophotometer was done by the use of blank of buffer and methanol.

- Drug permeation: A modified device (modified Franz diffusion cell using epithelial tissues facial part of the sheep was taken from the slaughter house in Muscat and washed with $0.9 \%$ normal saline and used as a diffusion membrane) was used for evaluation of drug permeation. The membrane was tied to a specifically designed glass cylinder (open at both ends). $13 \mathrm{ml}$ Simulated tear fluid $\mathrm{pH} 6.8\left(\mathrm{NaCl} 8 \mathrm{~g}, \mathrm{Na}_{2} \mathrm{HPO}_{4}\right.$ $2.38 \mathrm{~g}, \mathrm{~K}_{2} \mathrm{HPO}_{4} 0.19 \mathrm{~g}$ and $1000 \mathrm{ml}$ distilled water and the $\mathrm{pH}$ adjusted using $\mathrm{H}_{3} \mathrm{PO}_{4}$ ) was used as the diffusion medium in the receptor chamber. $1 \mathrm{ml}$ of the formulation which was to be tested was added to the donor chamber with the help of a syringe. The donor surface of the membrane was constantly in contact with simulated tear fluid. Temperature of $37 \pm 0.5^{\circ} \mathrm{C}$ was maintained throughout the test. A magnetic stirrer provided continuous agitation. $1 \mathrm{~mL}$ of sample was withdrawn every 1 hour up to 6 hours and replaced with same volume of fresh simulated tear fluid to maintain constant volume. The withdrawn samples were diluted to $10 \mathrm{~mL}$ in a test-tube with the prepared buffer and analysed by UV spectrophotometer at $240 \mathrm{~nm}$.

- Antimicrobial activity: Among prepared formulations, the gel which showed sustained release from the in-vitro drug release study was taken as the optimized gel for microbiological study to determine the antibacterial properties of Azithromycin against gram positive bacteria namely Staphylococcus aureus, streptococcus, Escherichia coli, and gram negative bacteria Klebsiella and Proteus using cup-plate method. Fresh cultures of different strains of bacteria were prepared and bacteria were sub-cultured in the media at room temperature. Then with the help of sterile cork borer, cups of around $4 \mathrm{~mm}$ diameter were made on media and the developed formulation $(1 \mathrm{ml})$ was poured into the cups made and incubated for 24 hours at $37^{\circ} \mathrm{C}$. The zone of inhibition was measured after incubation.

\section{Results and Discussion}

Physical appearance

The gels formulated were clear, viscous enough and homogenous with few bubbles present in them.

\section{pH readings}

The $\mathrm{pH}$ of the formulated gels was satisfactory and in acceptable ranges for the ophthalmic drug delivery systems as shown by table 2 .

\begin{tabular}{|c|c|c|c|c|}
\hline \multicolumn{5}{|c|}{ pH readings } \\
\hline Gels & Reading 1 & Reading 2 & Reading 3 & Mean pH \pm SD \\
\hline Gel 1 & 6.85 & 6.83 & 6.81 & $6.83 \pm 0.02$ \\
\hline Gel 2 & 6.88 & 6.84 & 6.82 & $6.85 \pm 0.031$ \\
\hline Gel 3 & 6.80 & 6.80 & 6.84 & $6.81 \pm 0.023$ \\
\hline Gel 4 & 6.79 & 6.83 & 6.85 & $6.82 \pm 0.031$ \\
\hline Gel 5 & 6.83 & 6.84 & 6.80 & $6.82 \pm 0.021$ \\
\hline Gel 6 & 6.86 & 6.84 & 6.83 & $6.84 \pm 0.015$ \\
\hline Gel 7 & 6.90 & 6.86 & 6.89 & $6.88 \pm 0.021$ \\
\hline Gel 8 & 6.82 & 6.86 & 6.85 & $6.84 \pm 0.021$ \\
\hline Gel 9 & 6.79 & 6.82 & 6.85 & $6.82 \pm 0.03$ \\
\hline
\end{tabular}

Table 2: pH measurements of the gels.

Key: SD: Standard Deviation

\section{In vitro drug release}

Standard curve from release study

The curve was obtained by using different concentrations made from the stock solution of $20 \mu \mathrm{g} / \mathrm{ml}$. The absorbance was measured and results are shown in figure 1.

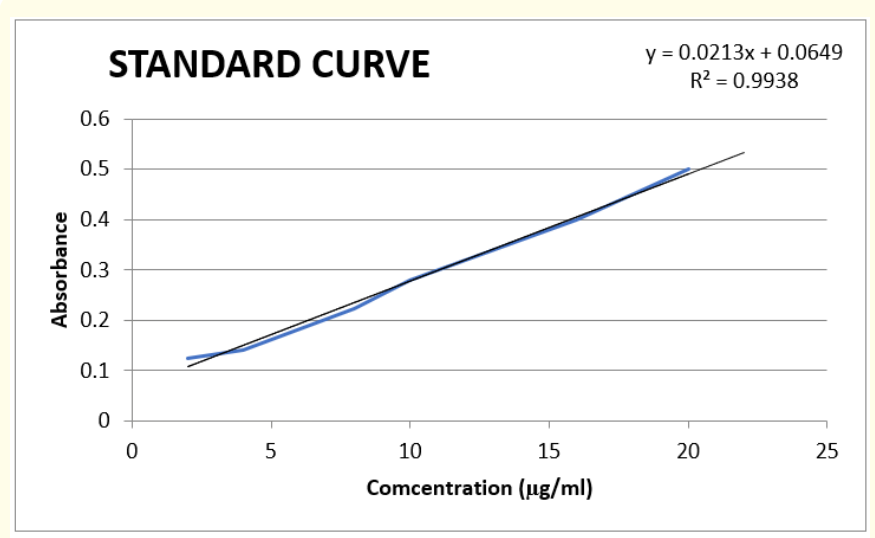

Figure 1: Standard curve for the drug.

\section{Release Characteristics}

The nine gel formulations of azithromycin were subjected to in vitro release studies for 6 hours and samples were taken over one hour interval. These in-vitro release studies were carried out using simulated tear fluid (STF) of $\mathrm{pH} 6.8$ as the dissolution medium using basket type dissolution apparatus. The cumulative percentages of drug released from each formulation are shown in table 3.

The drug release from formulated gels followed a linear pattern where the amount increased as the time increased from 1 to 6 hours. The drug was released more from the formulation containing $0.25(\% \mathrm{w} / \mathrm{w})$ of Carbopol 940. The release was 95, 98 and 90 from the Gel 2, Gel 1 and Gel 3 respectively. From these three gels nearly the entire drug was released after 6 hours. The same was demonstrated by Syed., et al. [16]. The drug release by the gels containing $0.375(\% \mathrm{w} / \mathrm{w})$ of Carbopol 940 were 76, 79 and 78 for the Gel 6, Gel 4 and Gel 5 respectively. The gels containing 0.5 (\%w/w) 


\begin{tabular}{|l|c|c|c|c|c|c|c|c|c|}
\hline $\begin{array}{c}\text { Time } \\
\text { (Hours) }\end{array}$ & $\begin{array}{c}\text { Gel 1 } \\
\text { \% DR }\end{array}$ & $\begin{array}{c}\text { Gel 2 } \\
\text { \% DR }\end{array}$ & $\begin{array}{c}\text { Gel 3 } \\
\text { \% DR }\end{array}$ & $\begin{array}{c}\text { Gel 4 } \\
\text { \% DR }\end{array}$ & $\begin{array}{c}\text { Gel 5 } \\
\text { \% DR }\end{array}$ & $\begin{array}{c}\text { Gel 6 } \\
\text { \% DR }\end{array}$ & $\begin{array}{c}\text { Gel 7 } \\
\text { \% DR }\end{array}$ & $\begin{array}{c}\text { Gel 8 } \\
\text { \% DR }\end{array}$ & $\begin{array}{c}\text { Gel 9 } \\
\text { \% DR }\end{array}$ \\
\hline 1 & 24.71 & 22.47 & 20.65 & 15.61 & 10.78 & 8.61 & 10.22 & 6.986 & 5.985 \\
\hline 2 & 64.12 & 59.78 & 57.47 & 23.87 & 20.86 & 19.18 & 14.7 & 11.48 & 11.48 \\
\hline 3 & 85.47 & 71.47 & 66.57 & 35.7 & 34.09 & 31.92 & 22.47 & 24.78 & 17.78 \\
\hline 4 & 91 & 79.24 & 74.34 & 57.47 & 54.88 & 52.64 & 37.52 & 45.36 & 36.47 \\
\hline 5 & 97.3 & 86.24 & 82.46 & 70.84 & 69.23 & 66.92 & 60.48 & 60.55 & 53.55 \\
\hline 6 & 98 & 94.78 & 89.74 & 79.38 & 77.84 & 75.95 & 77.14 & 72.24 & 66.78 \\
\hline
\end{tabular}

Table 3: Percent cumulative drug release (DR) from different formulations.

Key: \% DR indicates percentage of cumulative drug release.

of Carbopol 940 showed a percentage of release of 72, 67 and 77 for the Gel 8, Gel 9 and Gel 7 respectively.

Hence the percentage of drug release from the formulated gels followed the pattern, $0.25(\% \mathrm{w} / \mathrm{w})>0.375(\% \mathrm{w} / \mathrm{w})>0.5(\% \mathrm{w} / \mathrm{w})$ for the Carbopol concentrations. Gel 1 showed highest drug release whereas gel 9 showed delayed and sustained release of drug over 6 hours.

The higher amount of drug release was attributed to low concentration of the polymer which gave the drug chance to be easily released from the gel formulations. These results indicate that structure of the gel functioned as a resistant barrier to drug release as the concentration of polymers increased. On the basis of desired release study results Formulation 9 was taken as the optimised formulation for further studies.

Factorial design of percent release

Gel dissolution provides an indication of the release from the gel formulation. A decrease in release of the drug from gel formulation was seen as the concentration of the polymers increased (Figure 2). This may be due to formation of new bond between the Azithromycin and Carbopol 940 polymer which explained the reduction in release. The coefficient of correlation was good. The use of mucoadhesive polymers such as HPC and Carbopol 940 directly affect the gel release. Carbopol played a role in drug release while HPC was not having an effect in drug release which was proved by the study. However HPC might be helpful is preparing robust matrix.

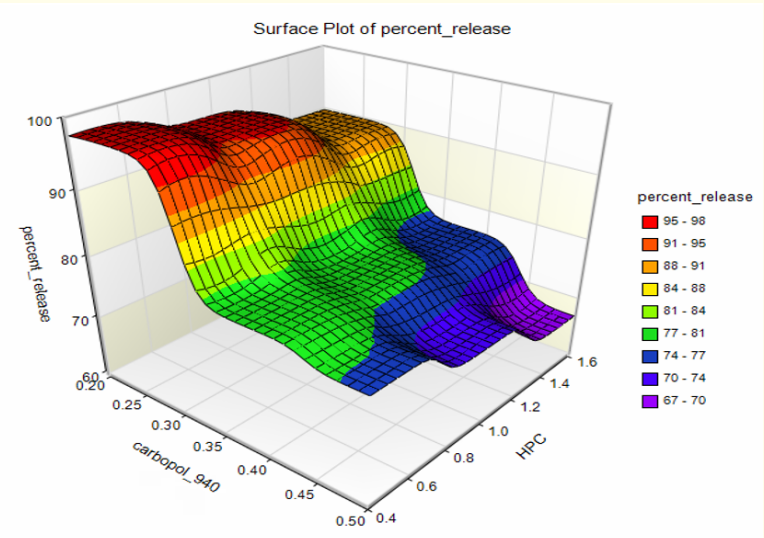

Figure 2: Effect of variables on percent drug release from Carbopol 940P gels.

\section{Standard curve for permeation study}

The curve (Figure 3) was obtained by preparing six concentrations made from the stock solution of $20 \mu \mathrm{g} / \mathrm{ml}$ and absorbance from UV spectrophotometer was measured at $240 \mathrm{~nm}$.

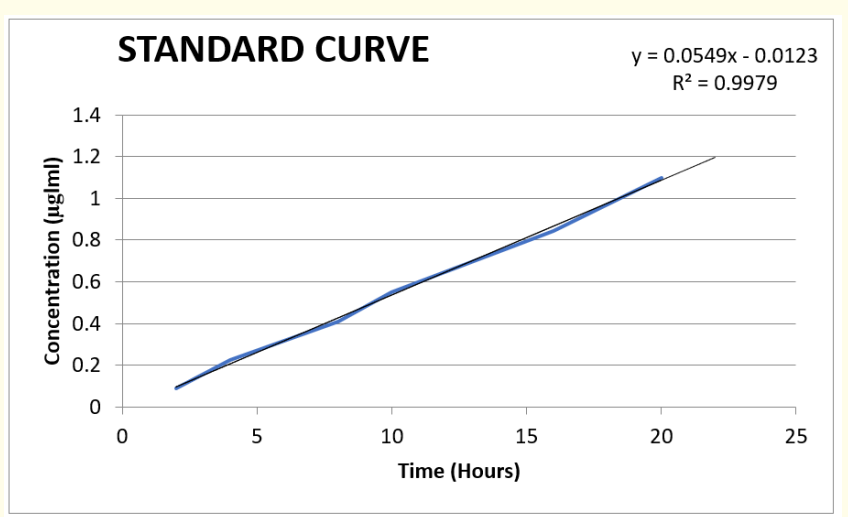

Figure 3: Standard curve for the drug.

\section{Diffusion study}

Using UV-Spectrophotometer, samples from permeation of Gel 9 were analysed and absorbance data was used to calculate concentration and amount of drug permeated across diffusion membrane. The results are shown in table 4

\begin{tabular}{|c|c|c|}
\hline \multicolumn{3}{|c|}{ Percent diffused drug } \\
\hline Time (Hr) & Concentration $(\boldsymbol{\mu g} / \mathbf{m l})$ & \% DD \\
\hline 1 & 10.3333 & 13.43329 \\
\hline 2 & 14.7778 & 19.21114 \\
\hline 3 & 24.8142 & 32.25846 \\
\hline 4 & 40.2969 & 52.38597 \\
\hline 5 & 57.6011 & 74.88143 \\
\hline 6 & 65.0692 & 84.58996 \\
\hline
\end{tabular}

Table 4: Percent cumulative drug diffused (\% DD).

Key : \% DD indicates cumulative percent of diffused drug through the epithelial cell.

From the data above, the amount of drug permeated after 1, 2, $3,4,5$ and 6 hours was 13, 19, 32, 52, 75 and $85 \%$ respectively. It seems that the amount of drug permeated followed linear pattern as the amount increased as the time increased. After 6 hours more than $80 \%$ of the drug permeated across the selected membrane 
barrier. This amount is somehow lesser than the amount released by the same gel in vitro using cellulose bag as a membrane barrier. This may be due to structural difference that exists in-vivo and in vitro but this amount is satisfactory and enough to suit good bioavailability of the formulation when used in the eye. The same was explained by Bhatia., et al. [17].

\section{Antibacterial test}

After incubation of media for 24 hours, the zone of inhibition (ZOI) was measured around the site where formulated optimized gel was placed. Diameter of zone of inhibition for each microorganism was measured to study their susceptibility against the drug Azithromycin. These values were compared with performance standards values of zone of inhibition to know whether the specific bacterium was resistant or sensitive to the drug, as represented in table 5 [18].

\begin{tabular}{|l|c|c|c|c|}
\hline \multirow{2}{*}{ Microorganisms } & \multirow{2}{*}{$\begin{array}{c}\text { Zone of } \\
\text { inhibition } \\
\text { (mm) }\end{array}$} & \multicolumn{3}{|c|}{$\begin{array}{c}\text { Performance } \\
\text { standards (mm) }\end{array}$} \\
\cline { 3 - 5 } & & Rs & I & S $\geq$ \\
\hline Staphylococcus aureus & 31 & 14 & $15-18$ & 19 \\
\hline Streptococcus spp. & 50 & 18 & $19-22$ & 23 \\
\hline Klebsiella spp. & 25 & 11 & $12-14$ & 15 \\
\hline Escherichia coli & 31 & 17 & $18-21$ & 22 \\
\hline Proteus spp. & 20 & 11 & $12-14$ & 15 \\
\hline
\end{tabular}

Table 5: Sensitivity test analysis.
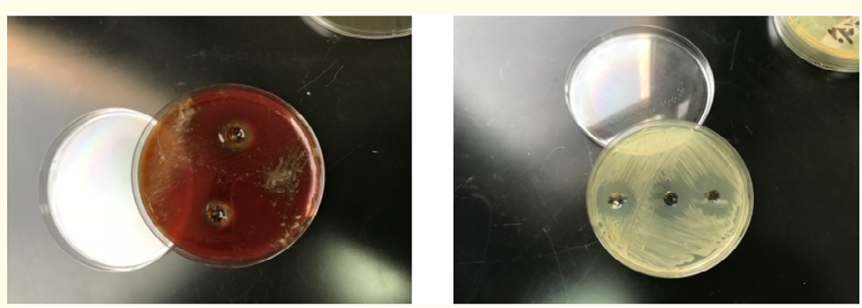

Figure 4: Sensitivity test.

The zones of inhibition obtained from the experiment were 31 $\mathrm{mm}, 50 \mathrm{~mm}, 25 \mathrm{~mm}, 31 \mathrm{~mm}$ and $20 \mathrm{~mm}$ for the organisms, Staphylococcus aureus, Streptococcus, Klebsiella, Escherichia coli and Proteus respectively. These zones of inhibition (Diameters) are all greater than the standard values for the zone of inhibition that specifies sensitivity of organism to the antibiotic azithromycin. From this observation it shows that all of the selected organisms for the test are sensitive to the prepared formula, and the diffusion of the drug from the selected gel (Gel 9) was good enough to allow the drug to inhibit the growth of the respective microorganism.

\section{Conclusion}

The purpose of the present research work was to develop the ocular pharmaceutical formulations which can overcome the problem related to the conventional dosage form by formulating gels using different polymers. The prepared formulations were clear, homogenous and viscous. They were shown to give required re- lease profile in invitro release study as the drug release decreased with increase in concentration of polymers with respect to time. $\mathrm{pH}$ of the formulated gels was satisfactory and in acceptable range for the ophthalmic drug delivery systems with a pH mean of $6.8 \pm 0.2$. Gel 1 showed a rapid drug release whereas Gel 9 showed delayed release of the drug after 6 hours. The immediate release of drug is attributed to low concentration of the polymer, which caused the drug to be easily released from the gel formulations. The structure of the gel functioned as a resistant barrier to drug release as the concentration of polymers increased.

Diffusion study of optimized formulation (Gel 9) indicated that most of drug in the gel permeated across the epithelial cell. Antimicrobial studies indicated the effectiveness of the formulation to different strains of bacteria.

\section{Bibliography}

1. Patil R and Kumar R. "In situ Gelling System Novel Approach for Ophthalmic Drug Delivery". World Journal of Pharmacy and Pharmaceutical Sciences 3.7 (2014): 423-440.

2. Lee VHL. "Precorneal, corneal and postcorneal factors In Ophthalmic Drug Delivery Systems". Drug and the Pharmaceutical Sciences Journal 58 (1993): 59-81.

3. Miyazaki S., et al. "In situ gelling xyloglucan formulations for sustained release ocular delivery of pilocarpine hydrochloride". Indian Journal of Pharmaceutical Sciences 229 (2001): 29-36.

4. Jain SP., et al. "In situ ophthalmic gel of ciprofloxacin hydrochloride for once a day sustained delivery". Drug Development and Industrial Pharmacy Journal 34.4 (2008): 445-452.

5. Ma WD., et al. "Pluronic (acrylic acid) copolymers as In situ gelling vehicle for ophthalmic drug delivery system". International Journal of Pharmaceutics 350 (2008): 247-256.

6. Al-Kassas RS and El-Khatib MM. "Ophthalmic controlled release in situ gelling systems for ciprofloxacin based on polymeric carriers". Drug Delivery 16.3 (2009): 145-152.

7. Gupta S and Vyas SP. "Carbopol-Chitosan Based pH Triggered in situ Gelling System for Ocular Delivery of Timolol Maleate". Sciatica Pharmaceutica 78.4 (2010): 959.

8. Padma PJ., et al. "Formulation and evaluation of in-situ ophthalmic gels diclofenac sodium". Journal of Chemical and Pharmaceutical Research 2.3 (2010): 528-535.

9. Kesavan K., et al. "Effect of Hydroxypropyl-pcyclodextrin on the Ocular Bioavailability of Dexamethasone from a pH Induced Mucoadhesive Hydrogel". Current Eye Research 36.10 (2011): 918-929.

10. Wu H., et al. "Design and evaluation of baicalin containing in situ pH-triggered gelling system for sustained ophthalmic drug delivery". International Journal of Pharmaceutics 410.1-2 (2011): 31-40. 
11. Piyush BP., et al. "Design and characterization of ofloxacin mucoadhesive in situ hydrogel". African Journal of Pharmacy and Pharmacology 6.23 (2012): 1644-1652.

12. Lokhande UR., et al. “Design and Development of pH-triggered in-situ gelling system of Ciprofloxacin". International Research Journal of Pharmacy 3.5 (2012): 418-422.

13. Nisha SG and Charyulu RN. "A study on stability and in-vivo drug release of Naphazoline and antazoline in-situ gelling systems for ocular delivery". International Journal of Pharma and Bio Sciences 4.1 (2013): 161-171.

14. Nnamani PO., et al. "Evaluation of Hydrogels Based on Poloxamer 407 and Polyacrylic Acids for Enhance Topical Activity of Gentamicin against Susceptible Infections". Tropical Journal of Pharmaceutical Research 13.9 (2014): 1385-1391.

15. Prachi S and Swatantra KS. "pH sensitive hydrogels of levofloxacin hemihydrates for ophthalmic drug delivery". World Journal of Pharmaceutical Research 3.2 (2014): 4003-4022.

16. Kostas A Kagkelaris., et al. "An eye for azithromycin: review of the literature". Therapeutic Advances in Ophthalmology (2018): 1-14.

17. Bhatia BH., et al. "Studies on thermoreversive mucoadhesive ophthalmic in situ gel of Azithromycin". Journal of Drug Delivery and Therapeutics 3.5 (2013): 106-109.

18. Bayeh Abera and Mulugeta Kibret. "Azithromycin, fluoroquinolone and chloramphenicol resistance of non-chlamydia conjunctival bacteria in rural community of Ethiopia". Indian Journal of Ophthalmology 62.2 (2014): 236-239.

\section{Assets from publication with us}

- Prompt Acknowledgement after receiving the article

- Thorough Double blinded peer review

- Rapid Publication

- Issue of Publication Certificate

- High visibility of your Published work

Website: $\underline{w w w . a c t a s c i e n t i f i c . c o m / ~}$

Submit Article: www.actascientific.com/submission.php Email us: editor@actascientific.com

Contact us: +919182824667 